\title{
Typ-2-Diabetes und Blutdruck
}

\section{Welchen Vorhersagewert haben die Blutdruckmessung in der Praxis und die einzelnen Komponenten des ambulanten Blutdruck-Monitorings (ABDM) zu Beginn und im weiteren Verlauf im Bezug auf die Entwicklung kardiovaskulärer Komplikationen bei Typ-2-Diabetikern?}

- Die Ergebnisse der ABDM korrelieren mit der Entwicklung von hypertensiven Organschäden, den kardiovaskulären Komplikationen und der Langzeitprognose besser als diejenigen der konventionellen Messung. Unklar ist aber immer noch, inwieweit die Werte, die zu unterschiedlichen Zeitpunkten gemessen und für bestimmte Perioden gemittelt wurden, zu Beginn und vor allem im weiteren Verlauf prognostisch von Bedeutung sind.

Bei 607 Typ-2-Diabetikern wurde der Blutdruck konventionell und mittels 48-h-Langzeitmessung mindestens jährlich - nach Therapieumstellungen auch häufiger - in einem durchschnittlichen Zeitraum von 5,4 Jahren gemessen. Nach Zufallskriterien wurden die Patienten angehalten, die gesamte antihypertensive Medikation nach dem Aufwachen oder zumindest einen Blutdrucksenker am Abend vor dem Schlafengehen einzunehmen.

Während dieser durchschnittlichen 5,4 Jahre kam es zu 107 kardiovaskulären Komplikationen. Der Blutdruck war naturgemäß bei Patienten mit Komplikationen höher als bei denjenigen ohne Komplikationen. Es zeigte sich, dass der systolische Blutdruck im Schlaf und der nächtliche Blutdruckabfall („dipping bzw. non-dipping“) als prognostische Faktoren dominieren. Denn wenn man bei den Analysen den systolischen Blutdruck im Schlaf berücksichtigte, verloren andere Komponenten des Blutdrucks wie Tageswerte, 48-h-Werte und konventionelle Messungen ihre Signifikanz. Aus therapeutischer Sicht am wichtigsten ist die Beobachtung, dass das Überleben ohne Komplikationen mit dem Abfall des systolischen Blutdrucks im Schlaf signifikant korreliert.

\section{Kommentar}

Die konventionelle Blutdruckmessung in der Praxis hat zahlreiche Mängel. Für die Diagnose Hypertonie ist diese Methode unzureichend. Deshalb fordern die neuen Empfehlungen des "National Institute for Clinical Excellence" (NICE) und der europäischen Gesellschaft für Hypertonie (ESH) vor Therapiebeginn Langzeitmessung und / oder Selbstmessung. Aus den vorgenannten Ergebnissen muss man folgern, dass für die Therapieüberwachung, zumindest von Diabetikern, vermutlich aber auch aller $\mathrm{Hy}$ pertoniker, am besten die Langzeitmessung geeignet ist. Denn nur sie allein kann den prognostisch bedeutsamen Blutdruck im schlaf ermitteln.

H. HOLZGREVE .

- Hermida RC, Ayala DE, Mojón A et al. Sleep-time blood pressure as a therapeutic target for cardiovascular risk reduction in type 2 diabetes. Am J Hypertens. 2012;25(3):325-34.
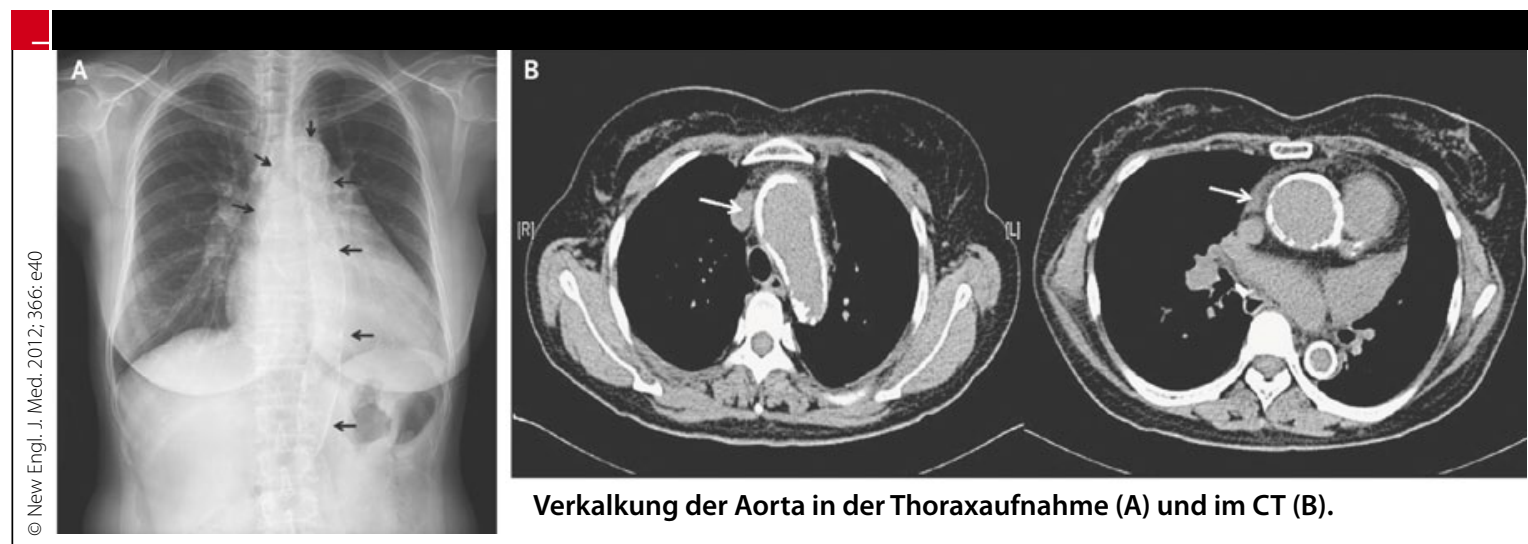

Verkalkung der Aorta in der Thoraxaufnahme (A) und im CT (B).

\section{Porzellan-Aorta}

Eine 55-jährige Frau wurde zum Aortenklappen-Ersatz wegen einer schweren Aorteninsuffizienz überwiesen. Auf der Thoraxaufnahme stellten sich eine kräftige lineare Kalzifikationen im Verlauf der thorakalen Aorta dar (Abb. A, Pfeile). Computertomografisch ließen sich diese Verkalkungslinien in der Wand der gesamten thorakalen und oberen abdo- minellen Aorta lokalisieren (Abb. B, Pfeil). Es handelt sich um eine so genannte Porzellan-Aorta. Aufgrund dieses Befundes konnte der Aortenklappenersatzes nicht in der üblichen Technik durchgeführt werden, bei der man die Aorta vorübergehend abklemmt, da mit einer Gefäßruptur aufgrund der Verkalkungen zu rechnen war. Man empfahl der Patientin einen transluminalen Aortenklappenersatz, was sie aber ablehnte.
Unter medikamentöser Therapie hält sich die Symptomatik in Grenzen. Die Patientin wurde weiterhin ambulant kontrolliert.

H. S. FÜESSL —

- M.-K. Kang. J.-W. Ha

(Yonsei University College of Medicine Seoul, South Korea; Korres.: homes78@naver.com): Porcelain Aorta. New Engl. J. Med. 2012; 366: e40 